\title{
Research on Optical Glasses Single-Grit Low Speed Scribing and Linear Impact Based on Sub-surface Crack
}

\author{
Jiang Chen ${ }^{1,2, a}$, Feihu Zhang ${ }^{1, b}$ \\ ${ }^{1}$ School of Mechanical and Electrical Engineering,Harbin Institute of Technology,Harbin \\ 150001, China \\ ${ }^{2}$ School of Light Industry, Harbin University of Commerce,Harbin 150028,China \\ achenjiang2004@126.com, \\ bzhangfh@hit.edu.cn
}

Keywords: optical glasses, low speed scribing, linear impact, sub-surface crack, shape

Abstract. The wear particle impact action on specimen is directly related to the shape and depth of sub-surface crack in the grinding process of optical glasses. Thus, the research on the optical glasses grinding wear particle dynamic impact effect is an important fundamental task to the field of optical manufacture. According to the influence law of the optical glasses grinding wear particle impact effect on the shape of sub-surface crack, this paper studies on optical glasses single-grit low speed scribing and linear impact. There are long cracks extending to both sides in the sub-surface crack which is formed in low speed scribing. The gap contour of low speed scribing specimen is indistinct. There are circumferential cracks in the sub-surface crack which is formed in linear impact. The gap contour of linear impact specimen is distinct. This paper studies on the sub-surface crack shape of single-grit low speed scribing and linear impact optical glasses specimen and specimen gap contour. The results showed that the single-grit wear particle dynamic impact effect on optical glasses specimen has important influence on sub-surface crack shape. The optical glasses grinding sub-surface crack is dynamic impact crack.

\section{Introduction}

The grinding process of brittle hard materials such as optical glasses will introduce sub-surface damage such as microcrack[1,2]. The sub-surface damage has a significant influence on the performance of optics system[3-5]. The prophase grinding of traditional large non spherical mirror processing is not precise enough. So the grinding processing has to retain a larger surface shape and sub-surface layer removal allowance, and removal allowance is removed by polishing process, the processing cycle is longer[6]. The research on the formation mechanism of hard brittle optical materials grinding sub-surface crack are one of the important basic scientific problems of optical manufacturing field.

Existing studies on optical glasses grinding mechanism and sub-surface crack is mainly based on indentation fracture mechanics and static loading conditions. The sub-surface crack of indentation fracture mechanics is composed of the median crack and transverse crack, as shown in Fig. 1. The high speed dynamic impact effect of grinding wheel in the grinding process is not considered in indentation fracture mechanics, that is, the influence of the wear particle speed is not considered.

The dynamic impact effect is manifested as impact load on the material surface caused by the disturbance in the medium gradually from the near to the distant spread out and the formation of stress wave[7]. All solid materials have inertia and deformation. When the impact load which is changed with time acts on solid materials, it is always a process of stress wave propagation reflection and interaction inside solid materials[8]. The problem of statics is just ignoring the inertia of deformable solid media. Short duration of impact load means that the material will have a high strain rate(generally in the range of $10^{1} \mathrm{~s}^{-1} \sim 10^{4} \mathrm{~s}^{-1}$ ), has a significant impact on the deformation and fracture behaviors and mechanism of material. The single-grit low speed scribing and linear impact experiment of optical glasses is carried by the ultra precision flying cut machine of $\mathrm{K} 9$ in this paper. 
This paper studies on sub-surface crack shape and specimen gap contour, analyzes the influence law of wear particle dynamic impact effect on sub-surface crack.

\section{Low speed scribing and linear impact experiment}

The main movement of the grinding wheel in the grinding process is the rotary motion of the grinding wheel. K9 optical glasses specimen either absence or presence feed motion, the cutting process of specimen is the state of the cutting depth and cutting speed change. In order to study the wear particle dynamic impact, avoid cutting depth and cutting speed changing influence on studying on sub-surface crack, considering the cutting depth and cutting velocity direction unchanged, this paper carries diamond single-grit straight and level low speed grinding and horizontal straight line impact experiment. By comparing the micro crack shape of $\mathrm{K} 9$ optical glasses material in two kinds of cutting modes, the dynamic impact of single-grit is studied. The diamond single abrasive particle straight and level low score and single diamond grit straight and level impact experimental is carried by independently developed by Harbin Institute of technology ultra precision flying cut machine. The diamond single grinding grain and as abrasive particle vector cone head part installs on the $630 \mathrm{~mm}$ horizontal rotary cutter, diamond single abrasive to approximate horizontal rectilinear motion acts low-speed grinding and impact on optical glasses specimen.

The flying cutter rotates at the $630 \mathrm{~mm}$ level radius of gyration, shown as Fig.1 and Fig.2. K9 optical glasses specimen is $20 \mathrm{~mm} \times 20 \mathrm{~mm} \times 10 \mathrm{~mm}$. When the grinding, the impact line segment and the radius of curvature of the grinding grain which act on optical glasses specimen are very small, wear particle motion track arc line segment can be approximated as horizontal linear motion. Using ultra precision cutting machine for linear and linear impact experiments, the results of the continuous change of grinding particle velocity direction in the grinding machine are solved. You can study in abrasive velocity magnitude and direction are the same conditions, the comparison K9 optical glasses specimen of single grinding grain straight and level low characterization and single diamond grit straight and level impact, two modes of action of sub-surface micro crack shape.

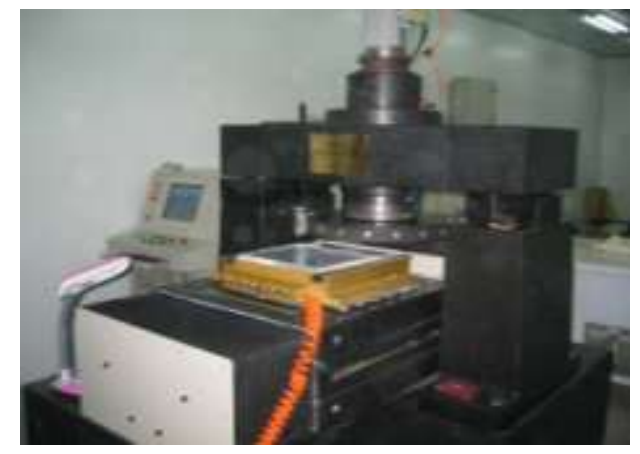

Fig.1 Ultra-precision fly cutting machine tool

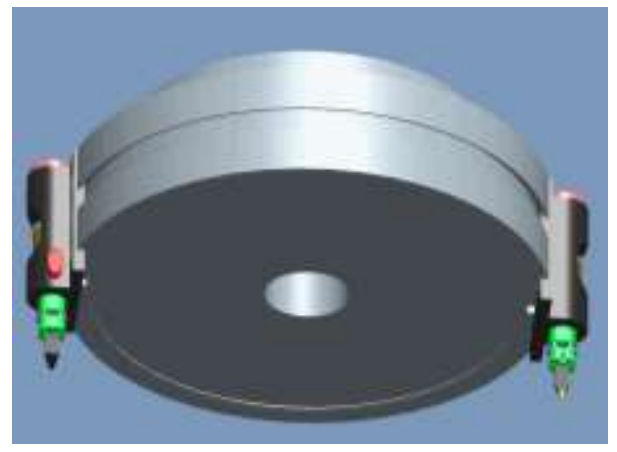

Fig.2 fly cutter

\section{Experiment on the low speed grinding of single-grit}

The single-grit and abrasive carrier parts are mounted on the cutter head of the ultra precision cutting machine tool, and the single-grit is cut from the surface of the optical glasses sample with a low speed uniform motion and $50 \mu \mathrm{m}$ cutting depth. In order to study the influence law of linear low speed grinding on the optical glasses sub-surface, avoid the stress complexity at the boundary of the specimen, the K9 optical glasses specimen $20 \times 20 \times 10 \mathrm{~mm}$ cross section polishing removal of $4 \mathrm{~mm}$ length is $20 \times 16 \times 10 \mathrm{~mm}$. Linear low ruling experiment is the essence of abrasive on the K9 optical glasses test of static compression fault removal, and the gap and crack / sub surface crack form and essence is the fracture results after abrasive and specimen material fully meet the static equilibrium conditions.

After ultra precision flying cutting machine tool linear slow and low speed scratching optical glasses specimen, the cross section with the vertical cross section of the surface scratch is polished, 
and the cross section is detected by SEM. Found that the outline of scratch notch is not clear, shell brittle fracture around the notch is obvious, there is a long crack in the presence of a scratch notch to the internal crack, shown as Fig.3.

\section{Optical glasses single-grit linear impact experiment}

K9 optical glasses single-grit impact experiment is carried by ultra precision cutting machine tool. The diamond single-grit with a certain speed, a certain depth of cut deep level linear impact optical glasses specimen. In order to eliminate the influence of boundary conditions on the stress state, the optical glasses specimen $20 \times 20 \times 10 \mathrm{~mm}$ after impact experiment is carried out to remove the $4 \mathrm{~mm}$ length, and the cross section was changed to $20 \times 16 \times 10 \mathrm{~mm}$. Then, the cross section is SEM detected, and the impact notch and micro crack shape are studied.

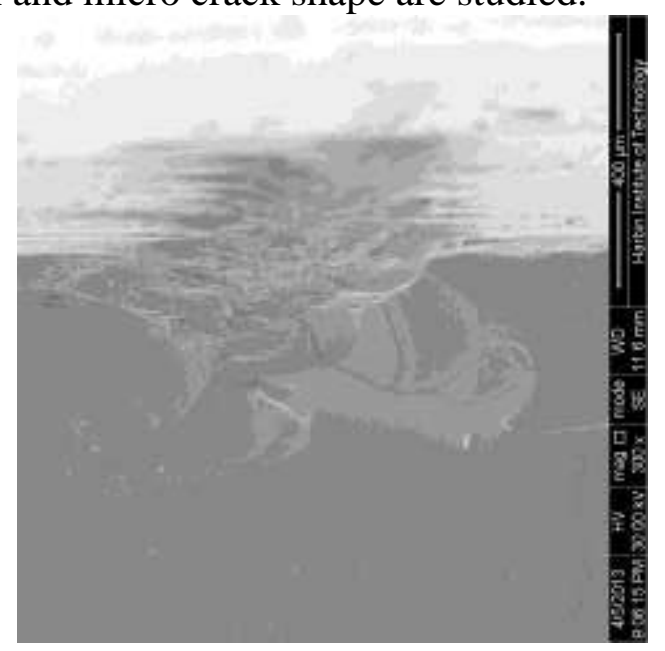

Fig.3 Micro crack shape of the scratch gap with single diamond grain grating the optical glass rectilinearly at lower speed

Will single diamond grit is installed on the $630 \mathrm{~mm}$ of the rotary cutter disc, cutting depth of $50 \mathrm{~m}$, a knife disc speed is $400 \mathrm{R}$ / min, a single diamond grit carries impact experiment on optical glasses specimen with horizontal linear motion.

When the diamond single-grit at a certain speed, a certain level of cutting depth impacts the optical glasses specimen, micro crack shape is shown as Fig.4. In the single-grit horizontal impact experiment, impact notch contour is clearly visible, long crack and extended internal crack are not obvious, exist circumferential crack.

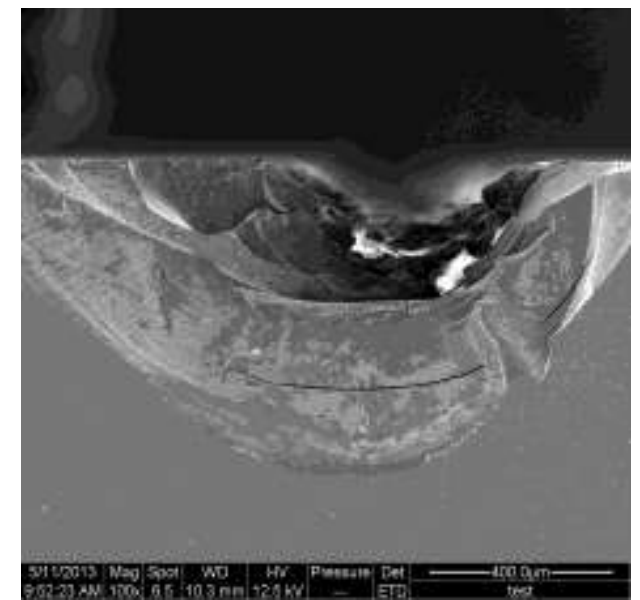

Fig.4 Micro crack shape of the impact gap with single diamond grain impacting the optical glass rectilinearly

\section{Analysis and discussion}

Comparative analysis horizontal slow speed linear grinding and horizontal linear impact experiment of single-grit, it is found that sub-surface micro crack shape are different at the section of K9 optical glasses specimen under two kinds of action modes of wear particle. The local brittle fracture 
destruction caused by the grinding particle level linear low velocity grinding experiment resulted in more massive shell fragmentation on both sides. Notch contour is not clear. There is a long micro crack which stretches to the inside and around. There isn' t circumferential crack. This is because of the low speed grinding meets the indentation fracture condition, the crack is indentation fracture mechanics crack.

Wear particle linear impact K9 optical glasses specimen notch contour is clear. The conchoidal crack is rarely seen at both sides of notch. The cracks which expend to the inside are less. The long crack is not obvious. There is circumferential crack. The experimental results from wear particle horizontal straight line low speed grinding and wear particle horizontal straight line impact shows that the impact velocity of single-grit has a significant influence on the formation of sub-surface crack shape of K9 optical glasses specimen. The shape and depth of the crack caused by the impact of abrasive particles on the K9 optical glasses specimen are different from the low speed grinding. Compared with the low speed grinding, the dynamic impact of the abrasive particles can reduce the depth of the sub-surface.

Under the action of wear particle impact load, the inner specimen material generates cylindrical surface stress wave. Propagation, diffusion and change law of stress wave is controlled by cylinder stress wave partial differential equations[9], as shown in formula (1) to type (6):

$$
\begin{gathered}
\rho_{0} \frac{\partial u}{\partial t}=\frac{\partial \sigma_{r}}{\partial r}+\frac{\partial \tau}{\partial z}+\frac{\sigma_{r}-\sigma_{\theta}}{r} \\
\rho_{0} \frac{\partial v}{\partial t}=\frac{\partial \tau}{\partial r}+\frac{\partial \sigma_{z}}{\partial z}+\frac{\tau}{r} \\
\frac{1}{\rho_{0} c_{1}^{2}} \frac{\partial \sigma_{r}}{\partial t}=\beta \frac{\partial u}{\partial r}+\alpha \frac{\partial v}{\partial z}+\alpha \frac{u}{r} \\
\frac{1}{\rho_{0} c_{1}^{2}} \frac{\partial \sigma_{z}}{\partial t}=\alpha \frac{\partial u}{\partial r}+\beta \frac{\partial v}{\partial z}+\alpha \frac{u}{r} \\
\frac{1}{\rho_{0} c_{1}^{2}} \frac{\partial \sigma_{\theta}}{\partial t}=\alpha \frac{\partial u}{\partial r}+\alpha \frac{\partial v}{\partial z}+\beta \frac{u}{r} \\
\frac{1}{\rho_{0} c_{2}^{2}} \frac{\partial \tau}{\partial t}=\frac{\partial v}{\partial r}+\frac{\partial u}{\partial z}
\end{gathered}
$$

The circumferential crack which appears in the wear particle impact sub-surface crack is completely different from the circumferential crack appears in the indentation fracture mechanics. It is because that the shape of wear particle impact sub-surface crack is related to the stress wave propagation and the diffusion rule. The wave front of wear particle impact stress wave propagates outward and diffuses as cylindrical surface. So the shape of the circumferential crack appears on the wear particle impact sub-surface crack. The wear particle impact sub-surface crack is dynamic impact crack. The linear velocity of conventional optical glass grinding is higher than the wear particle impact velocity of this experiment, so the sub-surface crack of optical glasses grinding is a dynamic impact crack, rather than the indentation fracture mechanics crack.

\section{Conclusion}

The shape of sub-surface crack is studied by low speed grinding and linear impact experiment of optical glasses, and the following conclusions can be drawn from the shape of the crack.

The long crack which extends to both sides is found in sub-surface crack under low speed grinding. And Specimen notch profile is not clear. The crack is indentation fracture.

The crack of the sub surface crack caused by the straight line impact has a circumferential crack, and the notch profile of the straight line impact specimen is clear. 
Due to the existence of circumferential crack in the impact crack, the dynamic impact crack can be seen from the shape of the dynamic impact crack, which is different from the static indentation fracture mechanics.

The dynamic impact effect of single-grit on optical glasses specimen has an important influence on the shape of sub-surface crack. The optical glasses grinding sub-surface crack is dynamic impact crack.

\section{Acknowledgements}

This work was financially supported Project(51175126) by National Natural Science Foundation of China (NSFC),Project(2011CB013202)supported by the National Basic Research Program(973 Program) of China.

\section{Reference}

[1] Shengyi Li,Zhuo Wang,Yulie Wu,Yifan Dai:Journal of Mechanical Engineeringt. Vol. 45 (2009),p.192-198.In Chinese

[2] Wang Zhuo,Wu Yulie,Dai Yifan,Li Shengyi:Aviation Precision Manufacturing Technology.Vol.43(2007),p.1-5.In Chinese

[3] Dongjiang Wu,Xiansuo Cao,Qiangguo Wang,et al:Optics and Precision Engineering.Vol.15(2007),p.1721-1726.In Chinese

[4] Zhuo Wang,Yulie Wu,Yifan Dai,Shengyi Li,Xusheng Zhou:Optics and Precision Engineering.Vol.16(2008),p.16-21.In Chinese

[5] Zihua Dai,Yongwei Zhu,Jianbin Wang,et al:Optics and Precision Engineering.Vol.21(2013),p.287-293.In Chinese

[6] Feng Shi,Yifan Dai,Xiaoqiang Peng,et al:Optics and Precision Engineering.Vol.18(2010),p.162-168.In Chinese

[7] J.C.Lambropoulos, S.D.Jacobs, B.E.Gillman, et al:J. Am. Ceram. Soc.Vol.88 (2005),p.1127-1132.

[8] Lili Wang,in: Foundation of Stress Waves.National Defense Industry Press,Bei Jing,(2010), p.227-240.In Chinese

[9] Jiang Chen,Feihu Zhang, Hang Zhao:SPIE.Vol.8415(2012),p. 841515:1-6. 